BMJ Open

Diabetes

Research

\& Care

\section{Relationship between diabetic nephropathy and depression: a cross- sectional analysis using the Diabetes Study from the Center of Tokyo Women's Medical University (DIACET)}

To cite: Takasaki K,

Babazono T, Ishizawa $\mathrm{K}$, et al. Relationship between diabetic nephropathy and depression: a cross-sectional analysis using the Diabetes Study from the Center of Tokyo Women's Medical University (DIACET). BMJ Open Diabetes Research and Care 2016;4:e000310. doi:10.1136/bmjdrc-2016000310

- Additional material is available. To view please visit the journal (http://dx.doi.org/ 10.1136/bmjdrc-2016000310).

Received 16 August 2016 Revised 21 September 2016 Accepted 6 November 2016

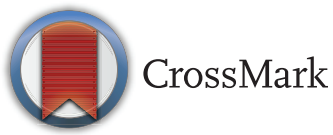

Department of Medicine, Diabetes Center, Tokyo Women's Medical University School of Medicine, Shinjuku-ku, Japan

Correspondence to Dr Tetsuya Babazono; babazono.dmc@twmu.ac.jp

Keiko Takasaki, Tetsuya Babazono, Kaya Ishizawa, Junnosuke Miura, Yasuko Uchigata

\section{ABSTRACT}

Objective: We conducted this cross-sectional study to investigate the relationship between stage of diabetic nephropathy and likelihood or severity of depression in patients with diabetes.

Research design and methods: We studied 2212 patients with diabetes (mean age 60.9 years; 928 women; 1838 patients with type 2 diabetes). Presence and severity of depression was examined using the Patient Health Questionnaire-9 (PHQ-9). Patients were classified into 5 stages of nephropathy, according to albuminuria and estimated glomerular filtration rate (eGFR); patients in stage 5 undergoing dialysis and kidney transplantation were analyzed separately (stages $5 \mathrm{D}$ and $5 \mathrm{~T}$ ). The relationship between stage of nephropathy and depression was examined using analysis of covariance and multivariate logistic regression analysis.

Results: Both least square mean PHQ-9 scores and prevalence of patients with $\mathrm{PHQ}-9$ scores $\geq 5$ points (mild depression) and $\geq 10$ points (moderate or severe depression) increased from stage 1 to $5 \mathrm{D}$, and then declined in stage 5T. Multivariate ORs for mild or greater depression increased in patients in stages 3, 4, and $5 \mathrm{D}$ in reference to those in stage 1 , which declined in patients in stage 5T. Albuminuria was significantly, but eGFR was not, associated with higher PHQ-9 scores and the PHQ- 9 scores $\geq 5$ or $\geq 10$ after adjustment for clinical findings.

Conclusions: In patients with diabetes, progression of nephropathy is likely to be associated with increased risk and severity of depression, which may be reduced after successful kidney transplantation. Albuminuria may be more strongly associated with depression than eGFR.

\section{INTRODUCTION}

Patients with diabetes frequently encounter depression, ${ }^{1}$ which may deteriorate glycemic control $^{2}$ and increase the risk of chronic diabetic complications. ${ }^{3}$ Similarly, patients with

\section{Significance of this study}

What is already known about this subject?

- Patients with diabetes frequently encounter depression, which may deteriorate glycemic control and increase the risk of chronic diabetic complications.

- Patients with chronic kidney disease also often experience depression, which has been associated with poorer outcomes.

What are the new findings?

- In Japanese patients with type 1 or type 2 diabetes, progression of stage of nephropathy including dialysis stage was associated with increased risk of depression and severity, as evaluated by Patient Health Questionnaire-9 (PHQ-9).

- The risk of depression reduced in patients undergoing kidney transplantation.

- Albuminuria was more strongly associated with depression than estimated glomerular filtration rate.

How might these results change the focus of research or clinical practice?

- Collaboration with psychiatrists may be essential for diabetologists to reduce the mental and physical burden of patients with diabetic nephropathy.

chronic kidney disease often experience depression, ${ }^{4}$ which has been associated with increased risk of rapid decline in glomerular filtration rate (GFR), reaching end-stage kidney disease (ESKD) and mortality after the start of dialysis. ${ }^{45}$ In contrast, patients who have undergone kidney transplantation have been shown to be less frequently associated with depression than those undergoing dialysis. ${ }^{6-8}$

Close relationships between diabetic nephropathy and depression are also observed. ${ }^{9-12}$ 
Cohort studies have demonstrated that depression in patients with diabetes leads to increased risk of ESKD. ${ }^{13}$ However, severity of depression has not been comprehensively examined among the stages of diabetic kidney disease. Moreover, the effects of kidney transplantation on depressive symptoms in patients with diabetes have not been well investigated. We therefore conducted this crosssectional study to clarify the relationship between stage of nephropathy and likelihood or severity of depression in patients with diabetes including those undergoing dialysis therapy and kidney transplantation. We also examined comparative effects of albuminuria and estimated GFR (eGFR), the two major manifestations of diabetic kidney disease, on depression symptoms.

\section{RESEARCH DESIGN AND METHODS \\ Subjects}

This was a single-center cross-sectional study, which was conducted as a part of the Diabetes Study from the Center of Tokyo Women's Medical University (DIACET), which was established in October 2012 by the DIACET committee (Chair: YU, Secretary: JM), as described previously. ${ }^{12}$ In the current study, among participants of the DIACET, we included Japanese patients with either type 1 or 2 diabetes (1) whose stage of diabetic kidney disease was identified according to laboratory data obtained within 90 days before and after distribution of the questionnaires, (2) those who had been treated with hemodialysis or peritoneal dialysis, and (3) those who had undergone kidney transplantation with a functioning graft at the time when the questionnaires were distributed. This study was conducted after receiving approval from the Ethics Committee of Tokyo Women's Medical University and obtaining informed consent from the participants.

\section{Patient Health Questionnaire}

Self-administered questionnaires asking health conditions, treatment status and medications, and comorbidities, as well as including items of the Patient Health Questionnaire-9 (PHQ-9) were distributed to patients at the time of their outpatient visit or admission. The questionnaires were retrieved either at the following outpatient visit or by mail and from inpatients during their hospitalization. The PHQ-9 comprises nine items inquiring about depressive symptoms. ${ }^{14}$ For each item, respondents choose one of the following responses with reference to the past 2 weeks: 'not at all', 'several days', 'more than half the days', or 'nearly every day'. These options correspond to scores of 0-3 points; the total score for all questions $(0-27)$ was calculated. On the basis of the total score of the nine questions, patients are classified into the following three levels of depression: no depression (0-4 points), mild depression (5-9 points), and moderate or severe depression (10 points or more).

In this study, patients with total scores above 5 and 10 were considered 'mild' and 'moderate-to-severe' depression, respectively. ${ }^{14}$ The reliability and validity of the Japanese version of the PHQ-9 was validated by Muramatsu et $a l^{15}$

\section{Definition of stage of diabetic nephropathy}

Stage of diabetic nephropathy was classified based on the revised classification proposed by the Joint Committee on Diabetic Nephropathy, consisting of the following four academic societies: the Japan Diabetes Society, the Japanese Society of Nephrology, the Japanese Society for Dialysis Therapy, and the Japan Society of Metabolism and Clinical Nutrition. ${ }^{16}$ Briefly, stage 1 was defined as normoalbuminuria $(<30 \mathrm{mg} / \mathrm{g}$ creatinine) and eGFR $\geq 30 \mathrm{~mL} / \mathrm{min} / 1.73 \mathrm{~m}^{2}$, stage 2 was defined as microalbuminuria $(30-299 \mathrm{mg} / \mathrm{g}$ creatinine) and eGFR $\geq 30 \mathrm{~mL} / \mathrm{min} / 1.73 \mathrm{~m}^{2}$, stage 3 was defined as macroalbuminuria $(\geq 300 \mathrm{mg} / \mathrm{g}$ creatinine $)$ and eGFR $\geq 30 \mathrm{~mL} / \mathrm{min} / 1.73 \mathrm{~m}^{2}$, stage 4 was defined as eGFR $<30 \mathrm{~mL} / \mathrm{min} / 1.73 \mathrm{~m}^{2}$ regardless of the urinary albumin level, and stage 5 was defined as patients currently undergoing dialysis or having a functioning kidney transplant.

In this study, patients in stage 5 undergoing dialysis and kidney transplantation were analyzed separately. The kidney transplant recipients were limited to those with a functioning kidney graft; therefore, patients who restarted dialysis therapy after losing kidney graft function were classified as patients on dialysis. Patients with type 1 diabetes who underwent simultaneous pancreas and kidney transplantation or pancreas transplantation after kidney transplantation were excluded, as pancreas transplantation may influence depressive symptoms and the number of patients was only six.

Serum and urinary creatinine levels were determined by the enzyme method, urinary albumin by immunonephelometry, and glycated hemoglobin (HbAlc) levels by high-performance liquid chromatography. eGFRs were calculated using the predictive equation for the Japanese proposed by the Japanese Society of Nephrology. ${ }^{17}$

\section{Other information related to diabetes}

The presence or absence and type of diabetes was confirmed by reviewing the medical records. The presence or absence of any stage of diabetic retinopathy, symptoms associated with peripheral polyneuropathy, and history of clinical visits for cardiac diseases, stroke, or gangrene were also asked in the questionnaire. ${ }^{12}$

\section{Statistical analysis}

Continuous data were expressed as an arithmetic mean and SD or geometric mean and $95 \% \mathrm{CI}$, with distribution range as required; categorical data were expressed by actual frequency and percentage. Continuous or categorical data among nephropathy stages were compared using Dunnett's or Dunn's multiple comparisons where stage 1 nephropathy was defined as the reference group. The Cochran-Armitage test was used to test an overall trend. 
PHQ-9 scores were compared among the nephropathy stage using analysis of covariance (ANCOVA) after adjustment for clinical findings, including age, gender, diabetes type, HbAlc levels, use of oral medications or injection drugs, presence or absence of diabetic retinopathy, symptoms associated with peripheral polyneuropathy, and history of clinical visits for cardiac diseases, stroke, or gangrene, as covariates. The ORs and $95 \%$ CIs for PHQ-9 scores above 5 or 10 according to nephropathy stage were calculated by multivariate logistic analysis after adjustment for the above clinical findings, defining stage 1 nephropathy as the reference group.

Finally, to compare the strength of the relationship with depression between albuminuria and eGFR in patients with stages 1-4 nephropathy, the area under the receiver-operating characteristic curve (AUROC) was compared using the method by DeLong ${ }^{18}$ among three models including either albuminuria or eGFR, or both albuminuria and eGFR.

All statistical analyses were performed using SAS/ STAT V.14.1 (SAS Institute, Cary, North Carolina, USA). Differences were considered statistically significant when $\mathrm{p}$ values were $<0.05$.

\section{RESULTS \\ Clinical characteristics of subject according to nephropathy stage}

Among 8128 individuals who completed the questionnaires between October 2012 and January 2013, individuals with normal or borderline glucose tolerance as well as patients with diabetes other than types 1 and 2 were excluded. Next, from the remaining 8058 patients with type 1 and 2 diabetes, as well as those with missing data on albuminuria, serum creatinine, or HbAlc levels within 90 days before and after the day the questionnaires were distributed $(\mathrm{N}=5841)$, those who did not answer more than one of the nine questions in the PHQ-9 ( $=314)$, and patients with type 1 diabetes who underwent simultaneous pancreas-kidney transplantation or pancreas transplantation after kidney transplantation $(\mathrm{N}=6)$ were eliminated. Finally, a total of 2212 individuals were included in this study (figure 1).

The mean $( \pm \mathrm{SD})$ age was $60.9 \pm 13.7$ years and the range was 30-92 years. There were 928 women and 1284 men, 374 patients with type 1 diabetes and 1838 patients with type 2 diabetes; 1297, 317, 120, 251, and 227 patients were classified as having stages 1-5 nephropathy, respectively. Among the patients with stage 5 nephropathy, 184 were undergoing dialysis and the remaining 43 had a functioning kidney graft. Clinical findings and laboratory data according to the stage of diabetic nephropathy are shown in table 1 . Patients with advanced stages of nephropathy were more likely to be men, old, treated with antidiabetic, antihypertensive, and antilipidemic mediations, and were also associated with increased diabetes duration and prevalence of diabetic complications. HbAlc levels were significantly higher in patients with stages 2 and 3 nephropathy but significantly lower in patients undergoing dialysis than in patients with stage 1 nephropathy.

The median dialysis duration in the 184 patients was 4.3 years (range 3 days to 27.5 years). Dialysis modality included hemodialysis in 138 patients, peritoneal dialysis in 9 patients, and combination of hemodialysis and peritoneal dialysis in 1 patient. The median postkidney transplantation time in the 43 kidney-transplanted patients was 4.4 years (range $0.5-21.4$ years).

\section{Comparison of PHQ-9 scores and depression frequency according to diabetic nephropathy stage}

Of the 2212 patients examined, 1387, 501, and 324 patients had total PHQ-9 scores of 0-4 points (no depression), 5-9 points (mild depression), and 10 points or more (moderate or severe depression), respectively. Figure 2 shows PHQ-9 score-based severity classification of depression according to the nephropathy stage. Both the proportions of patients with PHQ-9 scores $\geq 5$ points and those with PHQ-9 scores $\geq 10$ points increased in a stepwise manner from patients in stage 1 to the patients on dialysis in stage 5, and then declined in the kidneytransplanted patients in stage 5 (Cochran-Armitage test for the overall trend: $\mathrm{p}<0.001$ for both).

PHQ-9 scores after adjustment for age, gender, type of diabetes, HbAlc levels, medications, and presence of chronic diabetic complications by ANCOVA were significantly higher in patients in stage 4 and patients on dialysis in stage 5 than in those with stage 1 nephropathy (table 2). ORs for mild or greater depression, defined as PHQ-9 scores $\geq 5$ points, after adjustment for the demographic variables by multiple logistic regression analysis increased in a stepwise manner from patients in stage 2 nephropathy to patients on dialysis with statistical significance in those in stages 3 and 4, and patients on dialysis in reference to those in stage 1 , which declined in patients undergoing transplantation (table 3). Among the demographic data used as covariates in the multiple logistic regression analysis, those significantly associated with depression other than stage of nephropathy are also listed in table 3.

\section{Comparison of the effects of renal parameters on PHQ-9 scores}

To compare the strength of the association of albuminuria and eGFR with depression and its severity in patients with stages 1-4 nephropathy, we performed a multiple linear regression analysis using PHQ-9 scores as the dependent continuous variables, as well as a logistic regression analysis defining PHQ-9 scores $\geq 5$ or $\geq 10$ as dependent binary variables. Albuminuria was significantly associated with higher PHQ-9 scores and the scores $\geq 5$ or $\geq 10$ after adjustment for clinical findings, whereas eGFR was not associated with these outcomes (table 5).

Finally, receiver-operating characteristic curve analysis was conducted to compare predictability of presence 
Figure 1 Flow chart showing the number of study participants. DIACET, The Diabetes Study from the Center of Tokyo Women's Medical University; DM, diabetes mellitus; T1DM, type 1 diabetes mellitus; T2DM, type 2 diabetes mellitus; eGFR, estimated glomerular filtration rate; $\mathrm{HbA} 1 \mathrm{c}$, glycated hemoglobin; PHQ-9, Patient Health Questionnaire-9.

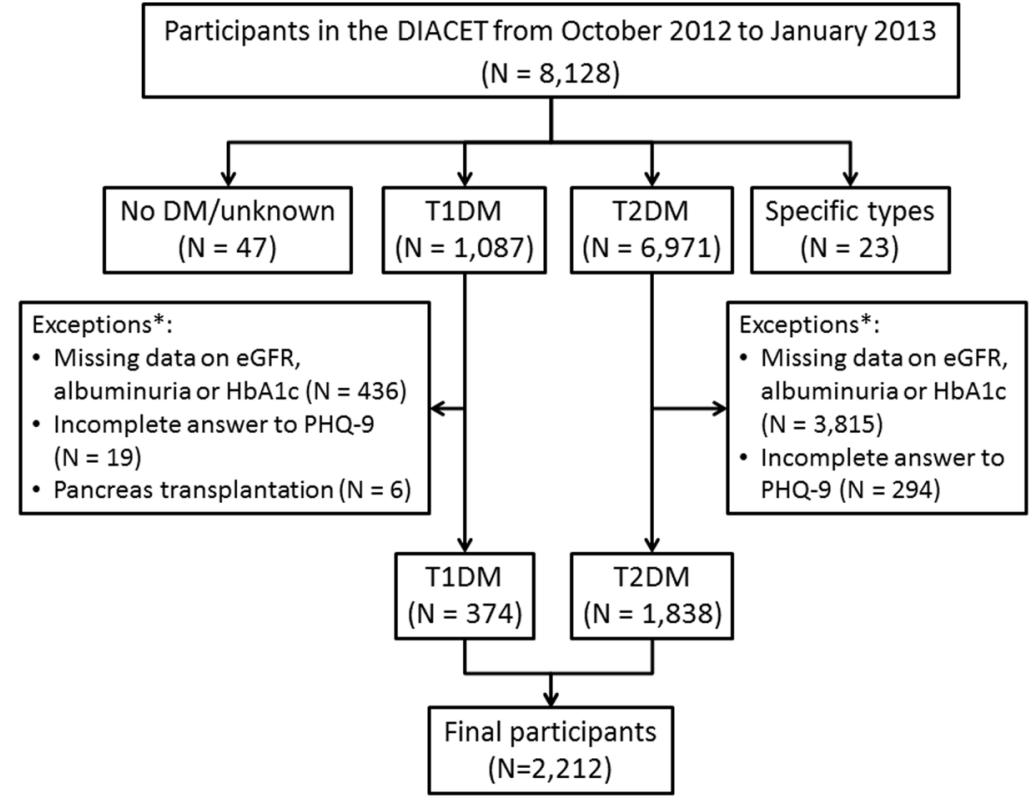

Table 1 Clinical characteristics and laboratory data in patients with diabetes classified by stage of nephropathy

\begin{tabular}{|c|c|c|c|c|c|c|}
\hline \multirow[b]{2}{*}{$\begin{array}{l}\text { Stage of diabetic } \\
\text { nephropathy }\end{array}$} & \multirow[b]{2}{*}{$1(\mathrm{~N}=1297)$} & \multirow[b]{2}{*}{$2(\mathrm{~N}=317)$} & \multirow[b]{2}{*}{$3(\mathrm{~N}=120)$} & \multirow[b]{2}{*}{$4(\mathrm{~N}=251)$} & \multicolumn{2}{|l|}{5} \\
\hline & & & & & $\begin{array}{l}\text { Dialysis } \\
(\mathrm{N}=184)\end{array}$ & $\begin{array}{l}\text { Kidney } \\
\text { transplantation } \\
(\mathrm{N}=43) \\
\end{array}$ \\
\hline Men (\%) & 52.8 & $65.6^{\star}$ & $68.3^{\star}$ & $62.2^{*}$ & $69.0^{*}$ & 60.5 \\
\hline Age (years) & $59.0 \pm 13.9$ & $64.0 \pm 12.2^{*}$ & $62.5 \pm 14.1^{*}$ & $66.7 \pm 13.3^{*}$ & $60.4 \pm 12.0$ & $56.2 \pm 9.2$ \\
\hline Type 1 diabetes (\%) & 21.7 & $8.5^{*}$ & 10.8 & $7.6^{\star}$ & 12.0 & 27.9 \\
\hline Diabetes duration (years) & $17.2 \pm 10.5$ & $19.5 \pm 10.9^{*}$ & $20.0 \pm 11.6^{\star}$ & $21.5 \pm 11.1^{\star}$ & $\begin{array}{l}23.8 \\
\pm 10.9^{*}\end{array}$ & $26.2 \pm 8.8^{*}$ \\
\hline Oral hypoglycemic agents (\%) & 61.5 & $74.5^{\star}$ & 70.0 & 57.4 & $49.5^{\star}$ & $30.2^{*}$ \\
\hline Injection agents (\%) & 48.3 & 48.9 & $71.7^{\star}$ & $71.7^{\star}$ & $69.6^{*}$ & $97.7^{*}$ \\
\hline Antihypertensive agents (\%) & 36.2 & $68.5^{\star}$ & $84.2^{*}$ & $81.7^{*}$ & 28.8 & $7.0^{\star}$ \\
\hline Antilipemic agents (\%) & 38.2 & 42.3 & 50.8 & $58.2^{\star}$ & $24.5^{\star}$ & $16.3^{\star}$ \\
\hline Retinopathy (\%) & 29.8 & $53.6^{\star}$ & $61.7^{*}$ & $69.7^{*}$ & $81.0^{*}$ & $83.7^{*}$ \\
\hline Neuropathy (\%) & 53.5 & $62.8^{\star}$ & $75.8^{*}$ & $78.9^{\star}$ & $78.0^{*}$ & 60.5 \\
\hline Cardiac disease (\%) & 10.3 & 16.7 & 20.0 & $25.9^{\star}$ & $32.1^{*}$ & 16.3 \\
\hline Stroke (\%) & 1.4 & 4.1 & 3.3 & 3.2 & 4.4 & 2.3 \\
\hline Gangrene (\%) & 0.4 & 1.3 & 3.3 & 2.0 & 4.9 & 9.3 \\
\hline $\mathrm{HbA1c}(\%)$ & $7.4 \pm 1.1$ & $7.7 \pm 1.2^{\star}$ & $8.0 \pm 1.6^{*}$ & $7.2 \pm 1.4$ & $7.1 \pm 1.3^{*}$ & $7.8 \pm 1.5$ \\
\hline Urinary ACR (mg/g) & $\begin{array}{l}7.9 \\
(7.6-8.2)\end{array}$ & $\begin{array}{l}81.5^{\star} \\
(75.6-87.9)\end{array}$ & $\begin{array}{l}912.6^{*} \\
\text { (784.5 to } 1061.8)\end{array}$ & $\begin{array}{l}631.8^{\star} \\
(558.6 \text { to } 958.7)\end{array}$ & NA & NA \\
\hline Serum creatinine (mg/dL) & $0.76 \pm 0.18$ & $0.90 \pm 0.26^{*}$ & $1.05 \pm 0.32^{\star}$ & $2.96 \pm 1.56^{\star}$ & NA & NA \\
\hline eGFR $\left(\mathrm{ml} / \mathrm{min} / 1.73 \mathrm{~m}^{2}\right)$ & $74.7 \pm 17.3$ & $65.5 \pm 19.6^{*}$ & $58.9 \pm 24.6^{*}$ & $19.6 \pm 7.3^{\star}$ & NA & NA \\
\hline
\end{tabular}

and severity of depression between albuminuria and eGFR. As shown in table 5, the areas under the curve (AUCs) predicting PHQ-9 scores $\geq 5$ and $\geq 10$ were significantly greater in the model of albuminuria than that of eGFR. Adding eGFR to the albuminuria model yielded no significant difference in the AUC.

\section{DISCUSSION}

In this single-center cross-sectional study of 2212 Japanese patients with type 1 or 2 diabetes, we have shown that later stages of nephropathy were incrementally associated with higher prevalence and more severe depression. In contrast, patients with a successful kidney 
Figure 2 Prevalence of depression severity according to the stage of diabetic nephropathy. The white bar shows both the number and proportion of participants who had total PHQ-9 scores of 0-4 points (no depression), the gray bar shows those who had 5-9 points (mild depression), and the black bar shows those who had 10 points or more (moderate or severe depression) in stages 1-5 undergoing dialysis and stage 5 undergoing kidney transplantation, respectively. PHQ-9, Patient Health Questionnaire-9.

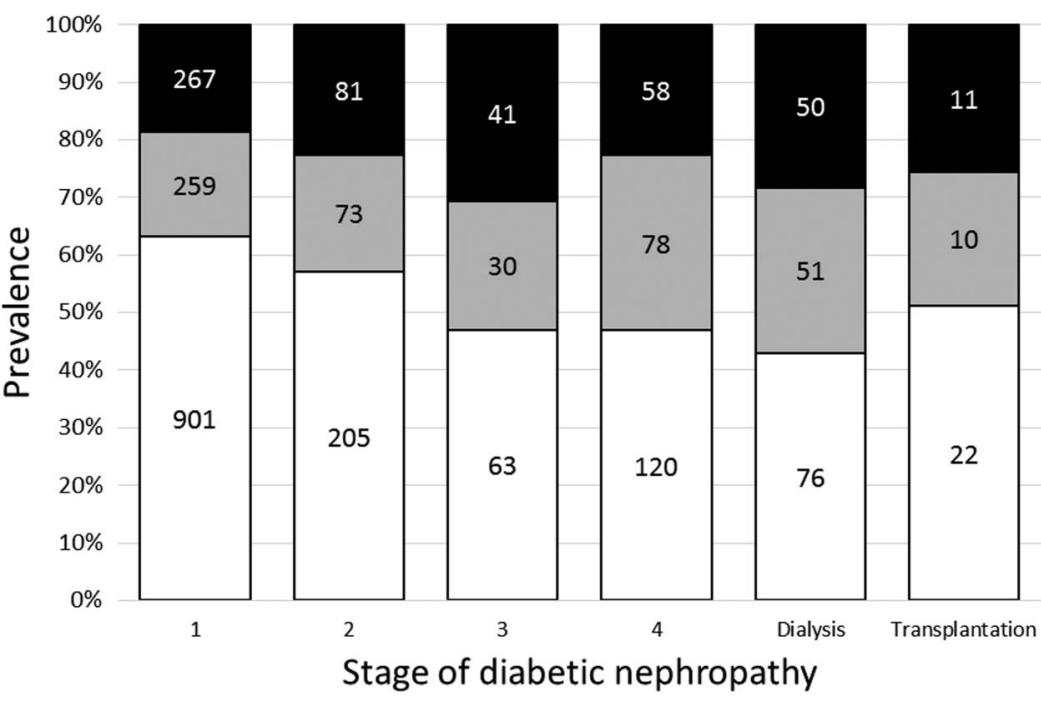

$\square$ No depression $\square$ Mild depression $\square$ Moderate or severe depression

Table 2 Adjusted PHQ-9 scores in patients with diabetes classified by stage of nephropathy

\begin{tabular}{|c|c|c|c|c|c|c|}
\hline \multirow[b]{2}{*}{ Stage of diabetic nephropathy } & \multicolumn{3}{|l|}{ Model 1} & \multicolumn{3}{|l|}{ Model 2} \\
\hline & $\begin{array}{l}\text { Least square } \\
\text { mean }\end{array}$ & $95 \% \mathrm{Cl}$ & p Value & $\begin{array}{l}\text { Least square } \\
\text { mean }\end{array}$ & $95 \% \mathrm{Cl}$ & p Value \\
\hline 1 (reference) & 3.85 & 3.59 to -4.11 & - & 4.00 & 3.74 to 4.25 & - \\
\hline 2 & 4.11 & 3.59 to 4.64 & 0.903 & 4.05 & 3.54 to 4.56 & 1.000 \\
\hline 3 & 5.64 & 4.79 to 6.49 & $<0.001$ & 5.12 & 4.29 to 5.94 & 0.063 \\
\hline 4 & 5.93 & 5.34 to 6.52 & $<0.001$ & 5.64 & 5.02 to 6.25 & $<0.001$ \\
\hline 5 (dialysis) & 7.02 & 6.33 to 7.71 & $<0.001$ & 6.59 & 5.79 to 7.39 & $<0.001$ \\
\hline 5 (transplantation) & 6.19 & 4.76 to 7.61 & 0.008 & 5.48 & 4.09 to 6.88 & 0.183 \\
\hline
\end{tabular}

Least square mean PHQ-9 scores were calculated using the analysis of covariance in patients with diabetes in each stage of nephropathy (stage 1 nephropathy as the control group) without adjustment (model 1) and with adjustment for age, sex, type of diabetes, HbA1c, use of oral hypoglycemic agents, injections, antihypertensive and antilipemic agents, presence of retinopathy, neuropathy, cardiac disease, stroke, and gangrene (model 2).

HbA1c, glycated hemoglobin; PHQ-9, Patient Health Questionnaire-9.

graft showed lower PHQ-9 scores and smaller risk of depression than those undergoing dialysis. These results were independent of other demographic variables that may affect depressive symptoms, suggesting that progression of diabetic nephropathy and therapeutic modality for ESKD per se are closely associated with the presence and severity of depression in patients with diabetes. We have also shown that albuminuria was more likely to be associated with depression than decreased eGFR; however, the absolute predictive accuracy of albuminuria was considered modest judging from the AUROC.

Several studies have shown a relationship between diabetic nephropathy and depression $;^{9-13}$ however, these studies simply observed the association of depression with the presence of diabetic nephropathy. Detailed information is therefore lacking regarding the relationship of depression with nephropathy stage, microalbuminuria, eGFR, and therapeutic modality for ESKD in patients with diabetes. Among the limited number of studies, the
Pathway Study consisting of 4082 US community-dwelling patients with diabetes demonstrated that PHQ-9 scores $\geq 10$ were associated with an up to 1.5 -fold increase in adjusted OR of albuminuria. ${ }^{10}$ In the Diabetes and Aging Study, an ancillary study to the Diabetes Study of Northern California (DISTANCE), the risk of depression defined as PHQ-8 scores $\geq 10$, in which the ninth question assessing suicidal or self-injurious thoughts was eliminated from PHQ-9, was high in patients with reduced eGFR among 5805 patients with diabetes aged 60 years or older. $^{11}$ Our results are in line with these studies; however, to the best of our knowledge, no previous studies have evaluated prevalence of depression, based on PHQ-9 scores or other items, in each nephropathy stage, including ESKD.

In general patients with ESKD, kidney transplantation has been demonstrated to be less likely associated with depression than chronic dialysis in cross-sectional ${ }^{6} 7$ and longitudinal studies; ${ }^{8}$ however, information has been 
Table 3 ORs for depression severity defined as PHQ-9 scores $\geq 5$ and $\geq 10$ in patients with diabetes classified by stage of nephropathy

\section{PHQ-9 scores $\geq 5$}

\section{Model 1}

OR $\quad 95 \% \mathrm{Cl}$

Stage of diabetic nephropathy

1 (reference)

2

3

4

5 (dialysis)

5 (transplantation)

Age (per 10 years)

Gender (men vs women)

$\mathrm{HbA1c}$ (per 1.0\%)

Type of diabetes (type 2 vs 1 )

Oral hypoglycemic agents (yes vs no)

Injections (yes vs no)

Antihypertensive agents (yes vs no)

Antilipemic agents (yes vs no)

Retinopathy (yes vs no)

Neuropathy (yes vs no)

Cardiac disease (yes vs no)

Stroke (yes vs no)

Gangrene (yes vs no)

1.00

$\begin{array}{ll}- & 1.00-\end{array}$

$1.24 \quad 0.96$ to $1.61 \quad 0.100$

$2.06 \quad 1.41$ to $3.00<0.001$

$2.48 \quad 1.89$ to $3.27<0.001$

3.232 .36 to $4.44<0.001$

$2.17 \quad 1.18$ to $4.00 \quad 0.013$

ORs were estimated using a multivariate logistic model with stage 1 diabetic nephropathy as the reference group.

HbA1c, glycated hemoglobin; PHQ-9, Patient Health Questionnaire-9. 


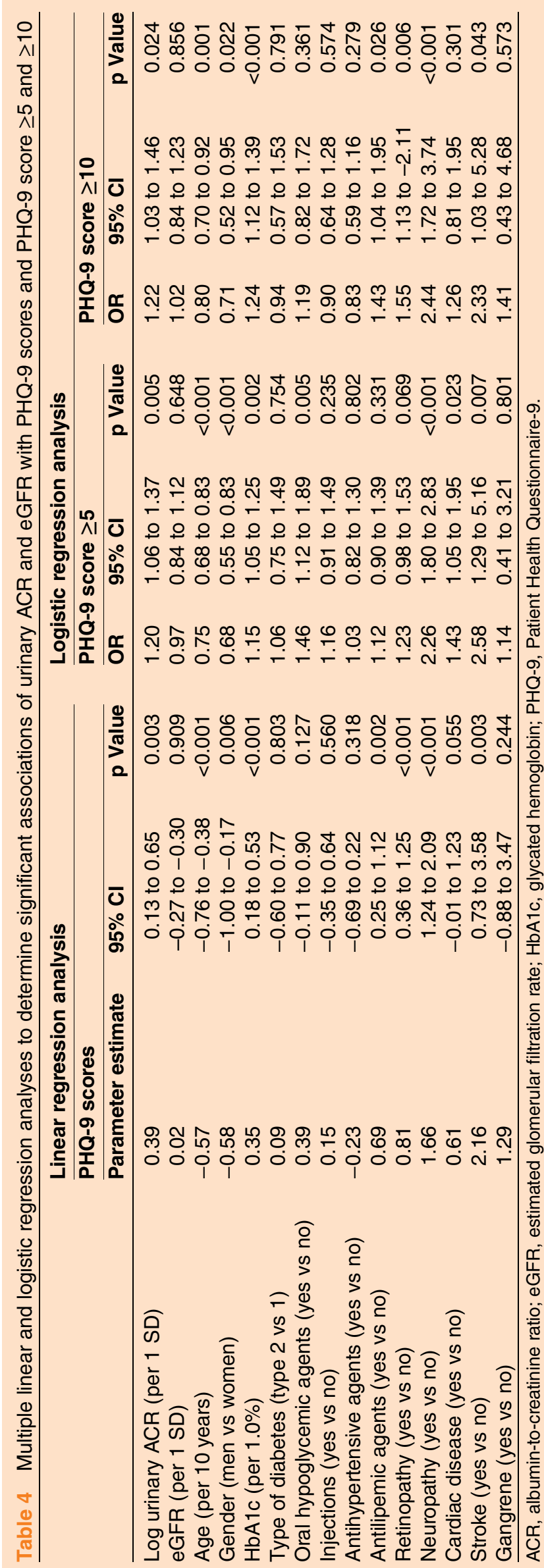

scarce in terms of the effects of kidney transplantation in patients with diabetes on depressive symptoms. This study suggested that risk of depression reduces after successful kidney transplantation also in patients with diabetes. Quality of life (QOL) has been repeatedly shown to be decreased in patients treated with either hemodialysis or peritoneal dialysis, leading to depressive symptoms. ${ }^{19}$ Therefore, mitigation of depressive symptoms after kidney transplantation as a result of improvement of QOL may be easily inferred. On the other hand, severe depression may decrease the patient's motivation for receiving a kidney transplant, ${ }^{20}$ which may have yielded a selection bias associated with lower prevalence of depression in kidney-transplanted patients in this study. Since this was a cross-sectional study, a longitudinal study over a period before and after kidney transplantation is required.

Similar to the Pathway Study, ${ }^{10}$ we found a significant association between albuminuria and depression, although unlike the DISTANCE survey, ${ }^{11}$ we found no significant association between reduced eGFR and depression. These cross-sectional studies, including ours, were unable to clarify the causal relationship of albuminuria and GFR with depression. Nevertheless, it is important to consider the potential mechanisms by which albuminuria and depression are mutually related. As previously reported, depression is bidirectionally associated with diabetes and its complications. ${ }^{122122}$ Thus, there are two potential mechanisms to consider: depression may lead to deterioration of glycemic control in patients with diabetes over a long period of time, which may be involved in onset of nephropathy. Alternatively, complication by nephropathy caused depression due to anxiety regarding future initiation of dialysis, or other reasons. ${ }^{23}$ Moreover, albuminuria is a known potential risk factor for onset of cardiovascular and cerebrovascular disorders through the cardiorenal link. ${ }^{24}$ We reported the influence of albuminuria to be stronger than that of eGFR in our previous cohort study. ${ }^{25}$ Hence, it is also conceivable that cardiovascular or cerebrovascular disorders had already complicated patients with advanced albuminuria, causing depression. ${ }^{26}$

Limitations of the methods and results of this study included its cross-sectional study design, preventing assessment of the causal relationship between the progression of nephropathy and the increased risk of depression; likewise, the causal relationship between kidney transplantation and reduced risk of depression. Moreover, since this study was conducted at a single university hospital, its findings cannot be generalized to all patients with diabetes. Selection bias also cannot be ruled out because participation in our DIACET study was voluntary, and fewer patients with depression may have participated in the study. We were unable to obtain data regarding other factors that influence depression including socioeconomical status, access to resources and support system, and uncomfortable symptoms. Finally, while the simplicity of the PHQ-9 is convenient 
Table 5 AUC of ROC curves for renal parameters predicting depression severity defined as PHQ-9 scores $\geq 5$ and 10

\begin{tabular}{|c|c|c|c|c|c|c|}
\hline \multirow[b]{2}{*}{ Renal parameter } & \multicolumn{3}{|c|}{ PHQ-9 scores $\geq 5$} & \multicolumn{3}{|c|}{ PHQ-9 scores $\geq 10$} \\
\hline & $\overline{A U C}$ & $95 \% \mathrm{Cl}$ & p Value* & $\overline{A U C}$ & $95 \% \mathrm{Cl}$ & p Value* \\
\hline Albuminuria & 0.574 & 0.546 to 0.601 & - & 0.573 & 0.532 to 0.614 & - \\
\hline eGFR & 0.518 & 0.489 to 0.546 & $0.001^{*}$ & 0.510 & 0.467 to 0.554 & $0.001^{*}$ \\
\hline Albuminuria and eGFR & 0.580 & 0.552 to 0.610 & 0.262 & 0.573 & 0.531 to 0.614 & 0.989 \\
\hline
\end{tabular}

AUC, area under the curve; eGFR, estimated glomerular filtration rate; PHQ-9, Patient Health Questionnaire-9; ROC, receiver-operating characteristic curve.

for diagnostic screening of depression in primary care and large-scale studies such as this study, it is known to lead to overdiagnosis of depression when it is used as a self-administered questionnaire. ${ }^{1}$ Future studies should examine this relationship using more accurate methods to diagnose depression, such as interviews with psychiatric specialists. Nevertheless, there has been no previous report of a detailed examination of this relationship based on nephropathy stage in more than 2000 patients with diabetes.

In conclusion, this study revealed the following findings in a large group of Japanese patients with type 1 or 2 diabetes: progression of stage of nephropathy including dialysis stage was significantly associated with risk of depression and the severity, as evaluated by use of PHQ-9; kidney transplantation was associated with reduced risk of depression compared with dialysis; and albuminuria was more strongly associated with depression than eGFR. Additional longitudinal studies are necessary to verify these causal relationships.

Acknowledgements The authors express their deep appreciation to the DIACET participants and the associated staff in the Department of Medicine and Ophthalmology, Diabetes Center, Tokyo Women's Medical University School of Medicine.

Contributors KT analyzed the data and wrote the manuscript. TB is the guarantor of this work and, as such, had full access to all the data in the study and takes responsibility for the integrity of the data and the accuracy of the data analysis. KI researched the data and contributed to the discussion. $\mathrm{JM}$ is the secretary and chief data administrator of the DIACET. YU contributed to the discussion and reviewed/edited the manuscript.

Competing interests DIACET is supported through an unrestricted research expenses from Alcon, Arkray, Astellas, AstraZeneca, Becton Dickinson, Boehringer Ingelheim, Chugai, Daiichi Sankyo, Eizai, Eli Lilly, Johnson \& Johnson, Kaken, KCl, Kissei, Kowa, Kyowa Hakko Kirin, Mitsubishi Tanabe, Mochida, MSD, Nipro, Novartis, Novo Nordisk, Ono, Otsuka, Pfizer, Roche, Sanofi, Santen, Sumitomo Dainippon, Takeda, Teijin, Terumo, and Torii.

Patient consent Obtained.

Ethics approval The Ethics Committee of Tokyo Women's Medical University.

Provenance and peer review Not commissioned; externally peer reviewed.

Data sharing statement No additional data are available.

Open Access This is an Open Access article distributed in accordance with the Creative Commons Attribution Non Commercial (CC BY-NC 4.0) license, which permits others to distribute, remix, adapt, build upon this work noncommercially, and license their derivative works on different terms, provided the original work is properly cited and the use is non-commercial. See: http:// creativecommons.org/licenses/by-nc/4.0/

\section{REFERENCES}

1. Anderson RJ, Clouse RE, Freedland KE, et al. The prevalence of comorbid depression in adults with diabetes: a meta-analysis. Diabetes Care 2001;24:1069-78.

2. Lustman PJ, Anderson RJ, Freedland KE, et al. Depression and poor glycemic control: a meta-analytic review of the literature. Diabetes Care 2000;23:934-42.

3. Lin EH, Rutter CM, Katon W, et al. Depression and advanced complications of diabetes: a prospective cohort study. Diabetes Care 2010;33:264-9.

4. Hedayati SS, Minhajuddin AT, Afshar M, et al. Association between major depressive episodes in patients with chronic kidney disease and initiation of dialysis, hospitalization, or death. JAMA 2010;303:1946-53.

5. Tsai YC, Chiu YW, Hung CC, et al. Association of symptoms of depression with progression of CKD. Am J Kidney Dis 2012;60:54-61.

6. Palmer S, Vecchio M, Craig JC, et al. Prevalence of depression in chronic kidney disease: systematic review and meta-analysis of observational studies. Kidney Int 2013;84:179-91.

7. Veater NL, East L. Exploring depression amongst kidney transplant recipients: a literature review. J Ren Care 2016;42:172-84.

8. González-De-Jesús LN, Sánchez-Román S, Morales-Buenrostro LE et al. Assessment of emotional distress in chronic kidney disease patients and kidney transplant recipients. Rev Invest Clin 2011;63:558-63.

9. de Groot M, Jacobson AM, Samson JA, et al. Glycemic control and major depression in patients with type 1 and type 2 diabetes mellitus. J Psychosom Res 1999;46:425-35.

10. Yu MK, Katon W, Young BA. Diabetes self-care, major depression, and chronic kidney disease in an outpatient diabetic population. Nephron Clin Pract 2013;124:106-12.

11. Campbell KH, Huang ES, Dale W, et al. Association between estimated GFR, health-related quality of life, and depression among older adults with diabetes: the diabetes and aging study. Am J Kidney Dis 2013;62:541-8.

12. Ishizawa K, Babazono T, Horiba $\mathrm{Y}$, et al. The relationship between depressive symptoms and diabetic complications in elderly patients with diabetes: analysis using the Diabetes Study from the Center of Tokyo Women's Medical University (DIACET). J Diabetes Complications 2016;30:597-602.

13. Yu MK, Weiss NS, Ding X, et al. Associations between depressive symptoms and incident ESRD in a diabetic cohort. Clin J Am Soc Nephrol 2014;9:920-8.

14. Kroenke K, Spitzer RL, Williams JB. The PHQ-9: validity of a brief depression severity measure. J Gen Intern Med 2001;16:606-13.

15. Muramatsu K, Miyaoka $\mathrm{H}$, Kamijima $\mathrm{K}$, et al. The patient health questionnaire, Japanese version: validity according to the mini-international neuropsychiatric interview-plus. Psychol Rep 2007;101:952-60.

16. Haneda M, Utsunomiya K, Koya D, et al. A new classification of diabetic nephropathy 2014: a report from joint committee on diabetic nephropathy. J Diabetes Investing 2015;6:242-6.

17. Matsuo S, Imai E, Horio M, et al. Revised equations for estimated GFR from serum creatinine in Japan. Am J Kidney Dis 2009;53:982-92.

18. DeLong ER, DeLong DM, Clarke-Pearson DL. Comparing the areas under two or more correlated receiver operating characteristic curves: a nonparametric approach. Biometrics 1988;44:837-45

19. Ginieri-Coccossis M, Theofilou P, Synodinou C, et al. Quality of life, mental health and health beliefs in haemodialysis and peritoneal dialysis patients: investigating differences in early and later years of current treatment. BMC Nephrol 2008;9:14.

20. Østhus TB, Preljevic V, Sandvik L, et al. Renal transplant acceptance status, health-related quality of life and depression in dialysis patients. J Ren Care 2012;8:98-106. 
21. Stewart JC, Rand KL, Muldoon MF, et al. A prospective evaluation of the directionality of the depression-inflammation relationship. Brain Behav Immun 2009;23:936-44.

22. Musselman DL, Betan $\mathrm{E}$, Larsen $\mathrm{H}$, et al. Relationship of depression to diabetes types 1 and 2: epidemiology, biology, and treatment. Biol Psychiatry 2003;54:317-29.

23. Collins MM, Corcoran P, Perry IJ. Anxiety and depression symptoms in patients with diabetes. Diabet Med 2009;26:153-61.

24. Anavekar NS, Gans DJ, Berl T, et al. Predictors of cardiovascular events in patients with type 2 diabetic nephropathy and hypertension: a case for albuminuria. Kidney Int Supp/ 2004;92: S50-5.

25. Bouchi R, Babazono T, Yoshida N, et al. Association of albuminuria and reduced estimated glomerular filtration rate with incident stroke and coronary artery disease in patients with type 2 diabetes. Hypertens Res 2010;33:1298-304.

26. Loomba RS, Aggarwal S, Arora R. Depressive symptom frequency and prevalence of cardiovascular diseases-analysis of patients in the National Health and Nutrition Examination Survey. Am J Ther 2015;22:382-7. 